\title{
The Social Side of Theory: A Community-Based Narrative of Research and Theory
}

\author{
Bernd Carsten Stahl, Jehad Al-Amri, Suad Almullah, \\ Muneeb Dawood, Christine Fidler, Mohanad Halaweh, \\ Osita Ibekwe, Raed Kareem Kanaan, Mick Phythian, \\ Abdullah Al-Shery, Khaled Swesi, and Sarai Tangai \\ De Montfort University, Leicester, UK
}

\author{
bstahl@dmu.ac.uk
}

\begin{abstract}
Theory is a central issue to be considered by researchers, particularly new researchers such as research students. In this paper we describe the role theory had in developing a research community. This community, the Information Society Doctoral Programme, faced with the usual questions that researchers have regarding theory, undertook a prolonged discourse on theory, using a range of means of discussion. The current paper describes the development of this discourse and its influence on the development of a shared research identity. The result of the discourse was not agreement on any particular theoretical positions but an increased ability to understand one another across theoretical divides. The paper thus develops a narrative of the role theory can have in shaping community, a perspective usually neglected in theoretical discourses.
\end{abstract}

Keywords: Theory selection and use, information society, social aspects research students, research community, discourse

\section{Introduction}

The concept of theory is central to any research but it has particular relevance to research students who are under a strong expectation to be conversant with theoretical issues in their field of study. Exposure to a variety of theories is, therefore, generally considered an important aspect of studying for a research degree. The emphasis of teaching theoretical matters tends to be placed on substantive theories that are considered relevant or on ways of acquiring knowledge about theory. One aspect that is much less frequently discussed is the social side of theory. It is often recognised that there are social arrangements (e.g. power structures in a research department) that influence which theory a student will consider, but it is less often recognised that debates concerning theory also hold the potential to contribute to the creation of communities and that such communities, in turn, have an influence on the

Material published as part of this publication, either on-line or in print, is copyrighted by the Informing Science Institute. Permission to make digital or paper copy of part or all of these works for personal or classroom use is granted without fee provided that the copies are not made or distributed for profit or commercial advantage AND that copies 1) bear this notice in full and 2) give the full citation on the first page. It is permissible to abstract these works so long as credit is given. To copy in all other cases or to republish or to post on a server or to redistribute to lists requires specific permission and payment of a fee. Contact Publisher@InformingScience.org to request redistribution permission. use of theory.

This paper concentrates on the relationship between theory and an emerging research community. We explore the role of theory in the constitution and development of collective research identities. The paper developed from an ongoing seminar series in a recently established doctoral programme at De Montfort University (Leicester, UK), called the Information 
Society Doctoral Programme (ISDP). This doctoral programme can be viewed as a research community. Such a research community does not develop independent of outside influences. An important influencing factor is constituted by academic issues, and central among them is the question of theory. This paper develops a narrative of the influence of discourses on theory on the development of our research community. In it we introduce the doctoral programme in more detail and describe the views and disagreements on theory. We contextualise these views on theory within the wider debate in Information Systems (IS) and academia.

\section{The Question of Theory of the Information Society}

Theory is similar to time, of which St. Augustine (354-430) said that he knows perfectly well what it is when nobody asks but it becomes completely unclear when required to define it. The same is true for theory: a concept we can easily use in a non-theoretical situation (i.e. a situation where one does not consciously reflect on it) but which is difficult to grasp when made an explicit object of reflection. It is certainly true that discussions of theory are an ongoing feature of most academic disciplines and possibly even more so in disciplines that lack a long history or strong institutional standing: disciplines such as IS. This may explain the continuing high level discussions of what theory is in the field of IS and how it can be 'used' (Gregor, 2006; Truex, Holmström, \& Keil, 2006).

While there is some uncertainty and debate surrounding theory by highly recognised scholars in areas such as IS, this uncertainty increases when one moves away from the recognised core of the discipline. This refers to the subject matter where questions of theory become more difficult to address when one works in several disciplines or across subject domains. It also raises questions for individuals who are new to the area or who have little experience in theoretical matters, as is typically the case for students. Questions of theory dominate the research project definition phase but they pose ongoing challenges throughout many Ph.D. projects. For these reasons, the question of theory took on a particular importance for us. In order to explain the specific issues we faced, we first outline the background situation and then explain how we tried to address the question of theory. This provides the setting for the discussion of findings, the contextualisation of the debate and its role in developing the community, and conclusions that resulted.

\section{The Information Society Doctoral Programme}

The Information Society Doctoral Programme (ISDP) was launched in spring 2006. Its aim was to allow us to focus the research supervision activities of those supervisors in the School of Computing who are interested in issues related to social and organisational use of information and communication technology (ICT). This includes scholars who are active in IS and consider themselves members of the IS community. The research interests of ISDP supervisors are not limited, however, to traditional IS topics. A majority of them are also members of the Centre for Computing and Social Responsibility (CCSR), a research centre of the School. As such they are interested in ethical and social consequences of ICT, which overlap partly with IS but which also branch off into other disciplines. The CCSR is a recognised and highly visible centre in the area of computer and information ethics but less visible in the mainstream IS community.

There were several reasons for launching the ISDP. One of them was that the number of Ph.D. applications was growing steadily and the ISDP represents an attempt to provide a clearer brand, which would allow potential students to identify a suitable place for research studies. In addition, there were internal political considerations. The creation of the ISDP helped strengthen the internal validity of our supervisory activities and, thereby, led to an internal identity that is important in negotiations for resources. There was also the aspect of the upcoming UK Research Assessment Exercise in 2008 (http://www.rae.ac.uk/), where the ISDP could be used to argue the point that research supervision is well organised. Furthermore, from the student viewpoint most impor- 
tantly, the ISDP was meant to provide an umbrella that would facilitate student interaction and would support the development of a research culture. Such a culture was felt to be highly beneficial to students who could profit from asking questions and exchanging ideas with their peers.

An added benefit of the growing research culture has to do with the student body of the ISDP. There are currently approximately 25 students: about half of them full time, the other half part time. The majority of our full time students are from the Arab world but we also have students with backgrounds from Africa, Asia, and America. Some, but not all, of these have gone through a prior degree in the UK. This means that our student body is highly diverse with very different academic and cultural backgrounds. Typical topics investigated by our students have to do with cultural, ethical, and social aspects of the use of ICT in society, e.g. in e-commerce or egovernment applications. Because of the diversity of student backgrounds and research interests, it was felt that a coherent research community was going to be a unique advantage as it offered a way of addressing many problems that go above and beyond the normal academic problems that research students face.

In addition to the above benefits, we also saw improved collaboration between supervisors as a main purpose of creating the ISDP. It is standard policy of De Montfort University to supervise students in teams of at least two supervisors. The practice of supervision varies considerably between faculties and research groups, as regulations only require that a minimum of one annual meeting of all members of the team takes place. A possible problem is that there may be very different styles of supervision and varying expectations among students. Creation of the ISDP meant that supervisors would also have the opportunity to develop a shared supervision culture, which would benefit the students by ensuring a more similar approach. One aspect of this shared culture is that within the ISDP it is now standard to have all supervision sessions attended by all (two or three) supervisors. The shared approach is, moreover, very useful in introducing new supervisors to current practices and allowing them to develop their skills as supervisors.

The ISDP students periodically engage in peer presentation of their work during our fortnightly research seminar and during progress report presentation of their research to graduate and undergraduate students in De Montfort University's Faculty of Computing Sciences and Engineering. Efforts are also being made to involve them in undergraduate tutorials, thus better preparing them for future teaching careers in academia as well as in other careers.

It was furthermore hoped that the new research culture, shared between students and staff, would lead to cross-fertilisation and thus to more and higher quality research outputs. By raising awareness of others' work, researchers can find shared interests and develop new research projects. Sharing of publication opportunities, conferences etc. should support this effect. As will become clearer below, the current paper can be seen as evidence of these activities occurring.

\section{ISDP and Theory}

The divergent backgrounds of our students mean that there is a wide range of research topics and approaches in the ISDP. A sign of this divergence of perspectives is the name of the "Information Society" Doctoral Programme. This title was chosen because it provides a broad and recognisable concept that arguably includes most IS research but it is also open to the issues of computer and information ethics that many of the ISDP members are interested in. In addition there is much non-academic work on the information society, much of it undertaken by international organisations. There is the information society discourse by the European Union but also the World Summit on the Information Society series under the auspices of the International Telecommunications Union and the United Nations Organization (UNO). At the same time it is sufficiently wide to allow for new developments and changing research questions. The term is ambiguous and it has been argued that the term is the result of a (mis)translation (Malaguerra, Niklowitz, \& Huber, 
2001). For our doctoral programme this meant that there is a lack of a clear literature tradition to build on and develop an academic identity.

The divergent backgrounds of the members of the ISDP can lead to problems of understanding, e.g. where mutual expectations between students and supervisors do not match. In order to address these issues and support the development of a research culture, we used several approaches simultaneously. The probably most important aspect of this was a research seminar. This seminar, which was timetabled for two hours every other week, was the main means for all members (staff and students) of the ISDP to meet and discuss. This started to run in October 2006 and is ongoing. The first session was used to set the agenda. It became clear that there were a number of issues that many of the students shared an interest in. Among these were questions of research approach and design, data collection and analysis, and, possibly most of all, issues of theory. We collectively set the agenda for the first year, trying to address as many of them as possible. Students were asked to take responsibility for individual sessions. These were typically prepared by reading a text and then discussing it in different ways throughout the seminar.

Additional means of facilitating collaboration in the ISDP are the use of a virtual learning environment (VLE), in our case the proprietary software called "Blackboard", which was mainly used to support the seminar series. Documents were made available on this VLE and all members of the ISDP had an opportunity to upload or download files of interest. In addition, a dedicated email listserv that included all members of the ISDP was set up.

\section{Links to other Discourses}

The setup of the ISDP as well as some of the questions we pursued will be familiar to most scholars working in comparable organisations. Seminars for research students are common and questions of theory tend to be a central issue discussed in them. The hopefully interesting aspect of our approach is that we not only discussed theory as an objective entity but that we went a step further and tried to reflect on the social nature and implications of theory. While there has long been a recognition of the social nature of theory and of its development in the philosophy of science (e.g. Kuhn, 1996) and related approaches, such as the sociology of science and technology (Latour \& Woolgar, 1979), little of this knowledge has filtered through to IS-related research.

This is not to say, however, that there are no related discourses that could inform our selfreflection. One important recent and ongoing debate in the area of IS, which has pertinence for questions of choice and justification of theory use, is the debate surrounding rigour versus relevance (Benbasat \& Weber, 1996; Benbasat \& Zmud, 1999, 2003; Ives, Parks, Porra, \& Silva, 2004). While the main point of contention in this debate is the question of the relationship of academic IS research to organisational IS practice, it has strong implications for the legitimacy of choice of theory. If IS is a field whose purpose it is to inform commercial practice, then theories have to be chosen in such a way that they are accessible to practitioners or at least the outcomes of research based on certain theories fulfil this criterion. If, on the other hand, IS is primarily an academic endeavour aiming to provide more convincing accounts of social phenomena involving some type of technology, then alternative theoretical approaches will be appropriate. Introna (2003) has shown that the rigour versus relevance debate can be seen as a struggle over the power to define acceptable discourses, which supports the assumption that such debates shape the perception and use of theory by new researchers who need to be perceived to be active in a legitimate field of research. Indeed, as we show below, such external debates have a strong bearing on what students see as suitable theories for their work.

There are further debates that we could draw from such as the debates in community informatics, information sciences, sociology of knowledge, critical theory of technology and many others. Many of these have implicitly influenced the current paper by inspiring some of the members of 
our community to think about theory in particular ways. We acknowledge that we are indebted to many scholars who have influenced our thinking and concede that it would lead beyond the confines of this paper to identify them all. More important than this attempt to trace the roots of our collective perception was our way of identifying what this collective perception was.

\section{Research Approach and Data Collection}

Towards the end of 2006 it was decided that we should explore what we mean by theory and whether there are shared aspects of theory that are applicable to all members of the ISDP. One aim was to help students develop their thinking with regards to theory. Another one was to find out whether there are substantial commonalities that would underpin a shared research culture. As a first step, all members of the ISDP were invited to write a brief, 2-page summary on their view of theory. They were asked for a short definition, for the theories they used, and for reasons for the choice of theory. The results were then discussed in the first seminar in 2007.

The discussion revealed that across the members of the ISDP there was wide variation in interpretation of the task. It also became clear that it would be difficult to discuss the issue of theory comprehensively within a two hour seminar. It was therefore decided to plan an away day where all members of the ISDP would be invited to spend a whole day discussing issues of theory. Funding for this day, which took place in May, 2007, was provided via the Faculty's Research Informed Teaching Initiative. During the away day, we started with a plenary discussion, which was used to define individual topics of interests that were subsequently addressed by four working groups. These groups took notes of their discussion, which were fed back to the group towards the end of the day. A main result of the day was the decision to write a collective paper on theory that would reflect the differences and agreements on the topic within the ISDP. Every member was invited to contribute and had a chance to revise the paper.

The entire process led to a significant number of documents from different authors; some were first person narratives, some were discussion minutes, and some were workgroup results. The process of developing reflective awareness of theory that this paper describes could thus draw on empirical material. One could also liken the approach to a number of established research approaches. In a general sense, the paper can be seen as a collective autoethnography where the authors are at the same time the subjects of study. There is an element of action research (Baskerville, 2001; Mumford, 2001) given that we have gone through several cycles of observation, planning, and action and that the entire exercise was meant to improve our practice. Despite this affinity to action research, we do not claim this to be an action research project, given that some aspects, notably the intervention, were not present. In terms of data collection and data analysis it can be described as an interpretive approach relying on the collection of qualitative data and emphasising the role of the researcher as part of the research outcome.

In the following discussion will draw on some of the ideas developed in our discourse. Quotes are printed in italics. We do not attribute the quotes to individuals because in many cases they are the result of minute taking, discussion, and collaboration and we wish to draw attention to the collaborative construction of our view of theory, which would be counteracted by individual attributions.

\section{Theoretical Discourse}

The result of our discourse on theory within the ISDP reflected many of the topics that are already established in the philosophy of IS debates. Such established results include the role of theory in the choice of research topics, the political nature of theory in ongoing power struggles in institutions, the relationship of research question and theory, and many others (Gregor, 2006; Truex et al., 2006). This is hardly surprising given that most of us are engaged as scholars in 
these debates and are at least aware of them and read the contributions. However, there were some surprises that arose from our attempt to find out whether there is a shared view of theory in the ISDP.

The first surprising issue is that the literature on theory in IS does not explicitly discuss the problem of uncertainty in recognising theory. Many of the less experienced researchers not only struggled with "using" or "applying" theory but got stuck at an earlier stage, where they had to identify a theory to use or apply. The statement I always find it difficult to distinguish between what is theory and what is not theory was typical for the view of several of the ISDP members. A related issue was the problem of finding out whether what is labelled theory is really theory. This seems to be a fundamental problem that is currently not sufficiently explored.

We tried to find an answer to this question by looking at definitions of theory. These were discussed extensively but it turned out that there was no agreement. Members of the ISDP used a range of definitions of theory and these were not always compatible. Among them could be found: a collection of concepts that are related to the discipline; an idea or concept that can be tested and applied; a set of conclusions arrived at through long research incorporating different research methods; a system of ideas that may have to be provable. Apart from these rather abstract views of theory, there was also the viewpoint that 'theory' in the context of Information Society research [is] a means of making sense of a given situation. We also discussed which theories people were using explicitly, which produced a long list including Technology Acceptance Model, Diffusion of Innovation, Theory of Reasoned Action, Motivation Theory, Information/Communication Theory, Systems Theory, Decision and Decision Making Theory, Chaos Theory, Critical Theory (Habermas), Structuration Theory, Evolutionary Theory, Actor Network Theory, and Quantum Theory.

In order to explain why researchers choose a particular theory, it appears to be important to consider the relationship between the researcher and the theory. In our investigation there were clearly two strands of debate visible: one that took an abstract and detached view of theory and another that was more conscious of the role of the individual researcher. A good example of the objectivist views theory would be: To me a theory is a concept which has been tested or argued for in a scholarly fashion; a concept which is famous enough within its own field to be mentioned very often in journals and books.

While this to some degree confirms positivist views on theory as an objective description of reality, which is meant to allow for description, simplification, and prediction, we were quite clear that, even from this point of view, theory had further purposes such as being protective and creating belonging.

Further investigation focusing on specific student concerns identified four main factors that influenced their choice of theory as indicated in Figure 1:

\section{Reliability}

One important influencing factor was the reliability of theory. Reliability is related to the trust in a theory. Trust can also be problematic for the research process unless it can be justified. One of researcher stated that:

If the theory is recommended by scholars in this field, I will go with it.

In this case reliability is considered to be one of the key issues that affect the decision for using a particular theory. 


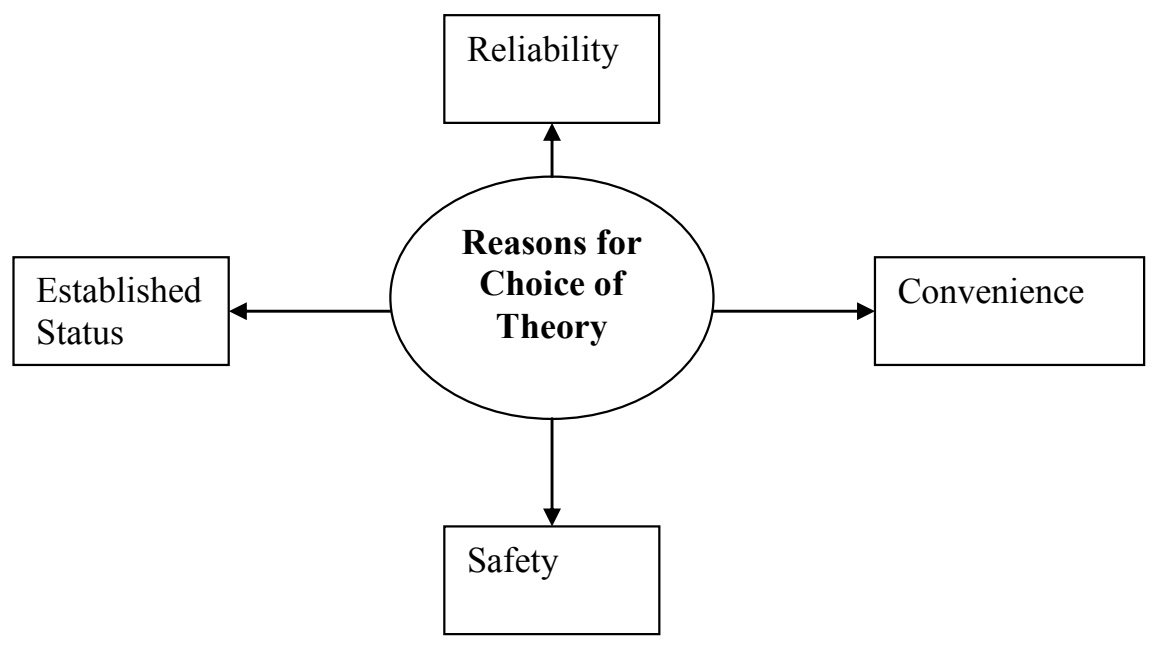

Figure 1: Factors influencing student choice of theory

\section{Established Status}

Directly related to the first point is the status that a theory enjoys in the discipline. Once a theory has been used many times in particular field of knowledge and has been tested empirically the researchers will be more confident to use this theory in their research. One of Ph.D. student's comments on this issue was:

I will give an example ... the TAM model in IS research is used by hundreds of studies, so it is worth to build on.

Using well established theories nevertheless raises the question whether they are applicable in a different context (e.g. different language, culture, technical application). It has to do with questions of validity and relates to research methodology.

\section{Safety}

One reason why students may prefer reliable and well established theories has to do with their perception of safety. Given the substantial investment in time and money that a $\mathrm{Ph}$.D. represents, many students are understandably risk averse and prefer to use theories they perceive as safe.

I prefer theory that can lead me to get my degree ... that is credible from literature review and can take me to the safe side.

\section{Convenience}

Again, linked to the pragmatics of undertaking a research project, students pointed out that convenience is an important factor. This refers to the availability of knowledge of a theory but, also, to further implication of theory. If the use of a particular theory promises straightforward data collection whereas an alternative theory would require more of an effort or the exploration of new techniques, then considerations of convenience may dominate other criteria.

These four factors show that theory choice and use is influenced by issues that theory itself typically does not consider. In the course of our discussions it was stated that anyone undertaking 
research is, explicitly or implicitly, using 'theory'. This was meant as a general epistemological statement on the condition of being able to describe the world. There was, however, also a strong recognition of further factors that force scholars to use theory. Research groups and staff need to play a political 'game' in order to obtain funding, to get their work published, to gain peer recognition and so on. From the student viewpoint it appeared clear that, when doing a literature review, every article has got to present a theory. Apart from the obvious politics, however, theory was also recognised as a way to avoid having to engage with a body of literature on research philosophy that is too great to be digested in the framework of a project of limited duration, including $\mathrm{Ph} . \mathrm{D}$. projects.

Such views did bring us back to the question of what constitutes a theory in the first place and which theories are likely to fulfil their goal of creating the academic legitimacy that is sought. A problem here is the equivocation of the term. There are theories which seem to be the rocks of knowledge of our worlds, and some others merely ideas, some seem firmly established, but others are not, like the theory of flying saucers is, just a theory. The important aspect seems to be to recognise which theories are capable of achieving the required legitimacy by displaying familiarity with discourses that reviewers or examiners are likely to expect. Several categories were suggested to achieve this. It was pointed out that there appears to be at least the categories of research theory, theorising on results (theories you produce as a result), and social theory (theories about what society is or should be and ways of thinking about society). A different categorisation, which was taken up quickly in our debates was the distinction between grand theories and small theories. Grand Theories (or Theories with a capital G and T) are those that are easily recognisable and our list of used theories reflects a view of these. Small theories are theories-in-use, theories that the researcher develops from the data (as in Grounded Theory, where Grounded Theory itself probably classes as a Grand Theory). This distinction is not exact at all but it seemed to resonate with many members of the ISDP who discussed these questions.

\section{Some Tentative Answers}

Our discussion of theory did not lead either to a new and overarching redefinition of theory or to a clearly identifiable body of theory in information society research. This is probably not surprising given the extensive discussion of theory in IS and the prior and even more extensive discussion in the philosophy of science and epistemology. The process of trying to understand our own use of the concept nevertheless proved to be useful for us for several reasons. It gave us a better understanding of the politics and psychology of choice of theory and it contributed to our shared identity as a research group in two differing yet interrelated ways. These aspects will both be elaborated on in the following three subsections.

\section{Politics and Psychology of Theory}

The politics of theory are often discussed and there is little denial of their importance (Lee, 2004). This is particularly pertinent for Ph.D. students who are often thought to have little choice with regards to theory and are required to follow their supervisors' suggestions, leading them down a certain path to understanding and using theory. There is some truth to this. A student working in a strongly positivist mainstream IS department might find it difficult to get a critical ethnography accepted as worthwhile research. However, our experience suggests that one should not overestimate such mechanisms. Our research group can probably be described as the opposite of the strongly positivist setting with many of the members of staff and supervisors interested in ethical issues and their qualitative exploration. None of them has a strong record in positivist or quantitative work. If the politics of Ph.D. research thesis were correct, then one should expect that the ISDP students are led towards an interpretive, critical, and qualitative approach. This is not the 
case. Many of our students choose positivist and quantitative theories and approaches, sometimes against the advice of their supervisors.

This raises the question of additional influencing factors. During our discussions of the issue it became clear that there are underlying assumptions and beliefs about theory and research that go far beyond rational discussion. Theory needs to be seen in the context of research and a possible $\mathrm{Ph} . \mathrm{D}$. project. Students come to a Ph.D. programme with ideas of what it means to do research and what is expected of them in their personal and professional environment. Such preconceptions can be stronger than any rational discussion about theory. How such preconceptions are formed is a different question, well worthy of further research. These preconceptions can also be seen as a link between the psychology and politics of research. Questions of acceptability of research influence the researcher's estimate of the validity of a theory. The researcher is not likely to accept a theory as useful if there is a question whether a potential publication outlet or a future employer is unlikely to accept it. These are value judgments for which there is typically only anecdotal evidence, if there is any evidence at all. They turn out to be highly influential value judgments nevertheless.

\section{Theory and Identity}

One of the goals of the ISDP's embarking on a discussion of theory was to explore whether there are aspects of theory that we share or whether theory provides a common link between us. Again, no simple answer can be given. This is probably not surprising. Grand Theories as well as small theories are important, and it may be more important to discuss these to understand their roles than to engage with them on a substantial content level. One can view many theories in IS as fashions in Abrahamson's sense (1996) and empirical research suggests that this is an appropriate view. This is often seen as problematic as fashions can be signs and symbols that facilitate understanding. If this is so, then the discussion of fashionable theories serves to ensure that we are able to communicate with one another. In a different context, but one with relevance for many members of the ISDP, Ricoeur (1988) once said that ethics, understood as moral philosophy, never provides judgments from nothing but always gives a retrospective explanation of common moral convictions. It may be possible to extend this line of reasoning to theories more generally, certainly to social theories. The reason for discussing theory would then move away from a correct description of the world towards a discourse that seeks to synchronise views of the world.

On a content level we found little that all members of the ISDP have in common in terms of research theories. The different topics, ranging from e-government to computer ethics and from ecommerce to service management, seem to require vastly different theoretical edifices. But despite the varying views of theory, the very fact that we tried to be more explicit about it and exchange views has contributed to a better shared understanding. This is an important building block of a shared identity, which was one of the main aims we pursued when starting our considerations of discourse.

\section{Community-based Learning Process and Identity}

Reflecting on the discourse between ISDP research community members, it became evident that our improved shared understanding of theory was not the only factor contributing to the development of our research identity. Rather, the very process by which our shared understanding of theory was emerging was reinforcing the development of a holistic and identifiable research community. This process is an example of community-based learning in action. Despite our very different personal backgrounds, formal positions, and cultures, the prevailing ISDP organisation is flat and the prevailing culture is one of equality and a sense of community and teamwork. This is different from many other research environments, where the overall culture is dictated to a large degree by the "Professors in Charge". Instead, the community-based learning activity we 
undertook when considering what theory is was enriched by our community organisation and culture, and the very act of performing this learning activity served to strengthen the ISDP community research identity.

In short, the process of forming a shared understanding of theory was in fact influenced by the community-based learning nature at ISDP, and at the same time this nature was indeed tested and improved by this project of seeking a shared understanding of theory.

\section{Conclusion}

Theory is a fundamental concept of all research and it is of particular importance in a crossdisciplinary and multi-disciplinary field like IS where it is generally seen as acceptable to import theories from any field one deems suitable. In this paper we have described the attempt to come to a better understanding of theory in the Information Society Doctoral Programme.

We found that many of the external discourses have a strong influence on our perceptions. The paradigm debates and ongoing discussion of theory in IS influence our view of the topic. We nevertheless found that some of the ongoing topics of the theory debate were not reflected in our group. The idea that theories can be chosen rationally, either because the topic demands it or because the politics of the organisation are of a certain type, seems to be only tenable to a limited degree. There are outside influences and psychological factors that limit the ability to choose theory.

An important distinction, which may be of limited analytical value but which nevertheless proved to be accessible to most of us, was the one between Grand Theories and small theories. Where academic publications speak of theory, they often mean Grand Theory. Grand Theory is important to ensure compatibility of discourses. At the same time, daily research practice is much more driven by small theories: theories-in-use or unconscious theories. There is probably a link between all these that will require further investigation.

The most important aspect of our entire attempt to tackle the issue of theory may be that the content level discussion of theory is not of primary importance. Whether a particular interpretation of the Theory of Reasoned Action, the Theory of Communicative Action (Habermas, 1981) or Structuration Theory (Giddens, 1984) is correct is often of secondary importance. The very act of discussing these theories may be much more relevant. Certainly in a research student programme such as the ISDP the social coherence created by the debate of theory and the ability to understand what others are saying within a community-based learning environment is more likely to contribute to the successful execution of research projects than any substantive or Grand Theory. This statement is of course a theoretical statement which we would like to be known as the Theory of Theory Reflection.

\section{References}

Abrahamson, E. (1996). Management fashion. Academy of Management Review, 21(1), 254-285.

Augustine. (354-430). Confessions of St. Augustine. Book X, Chapter 27. (Outler, Trans.). Retrieved 30 July 2008 from http://www.leaderu.com/cyber/books/augconfessions/bk10.html\#BOOKXCHAPX

Baskerville, R. (2001). Conducting action research: High risk and high reward in theory and practice. In E. Trauth (Ed.), Qualitative research in IS: Issues and trends (pp. 192-217). Hershey, PA: Idea Group.

Benbasat, I., \& Weber, R. (1996). Research commentary: Rethinking "Diversity" in information systems research. Information Systems Research, 7(4), 389-399.

Benbasat, I., \& Zmud, R. W. (1999). Empirical research in information systems: The practice of relevance. MIS Quarterly, 23(1), 3-16. 
Benbasat, I., \& Zmud, R. W. (2003). The identity crisis within the IS discipline: Defending and communicating the discipline's core properties. MIS Quarterly, 27(2), 183-194.

Giddens, A. (1984). The constitution of society - Outline of the theory of structuration. Cambridge: Polity Press.

Gregor, S. (2006). The nature of theory in IS. MIS Quarterly, 30(3), 611-642.

Habermas, J. (1981). Theorie des kommunikativen Handelns [The theory of communicative action]. Frankfurt a. M.: Suhrkamp Verlag.

Introna, L. (2003). Disciplining information systems: Truth and its regimes. European Journal of Information Systems, 12, 235-240.

Ives, B., Parks, M. S., Porra, J., \& Silva, L. (2004). Phylogeny and power in the IS domain: A response to Benbasat and Zmud's call for returning to the IT artifact. Journal of the Association for Information Systems, 5(3), 108-124.

Kuhn, T. S. (1996). The structure of scientific revolutions (3rd ed.). Chicago and London: The University of Chicago Press.

Latour, B., \& Woolgar, S. (1979). Laboratory life: The social construction of scientific facts. Beverly Hills and London: Sage Publication.

Lee, A. S. (2004). Thinking about social theory and philosophy for information systems. In J. Mingers \& L. Willcocks (Eds.), Social theory and philosophy for Information Systems (pp. 1 - 26) Chichester: Wiley.

Malaguerra, C., Niklowitz, M., \& Huber, M. (2001). Monitoring the information society? Proceedings of the IAOS satellite Meeting on Statistics for the Information Society, Tokyo, Japan.

Mumford, E. (2001). Advice for an action researcher. Information Technology \& People, 14(1), 12-27.

Ricoeur, P. (1988). Le cercle de la demonstration [The circle of demonstration]. In P. Ricoeur (1991), Lectures 1 - Autour du politique (pp. 216-230), Paris: Seuil.

Truex, D., Holmström, J., \& Keil, M. (2006). Theorizing in information systems research: A reflexive analysis of the adaptation of theory in Information Systems research. Journal of the Association for Information Systems, 7(12), 797-821

\section{Biographies}

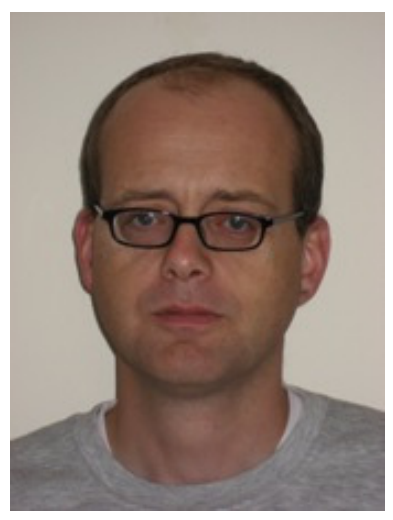

Bernd Carsten Stahl is a Reader in Critical Research in Technology in the Centre for Computing and Social Responsibility at De Montfort University, Leicester, UK. He coordinate's the Centre's Information Society Doctoral Programme. His interests cover philosophical issues arising from the intersections of business, technology, and information. This includes the ethics of computing and critical approaches to information systems. He is the Editor-in-Chief of the International Journal of Technology and Human Interaction.

Christine Fidler is Principal Lecturer and one of the supervisors of the Information Society Doctoral Programme (ISDP). 


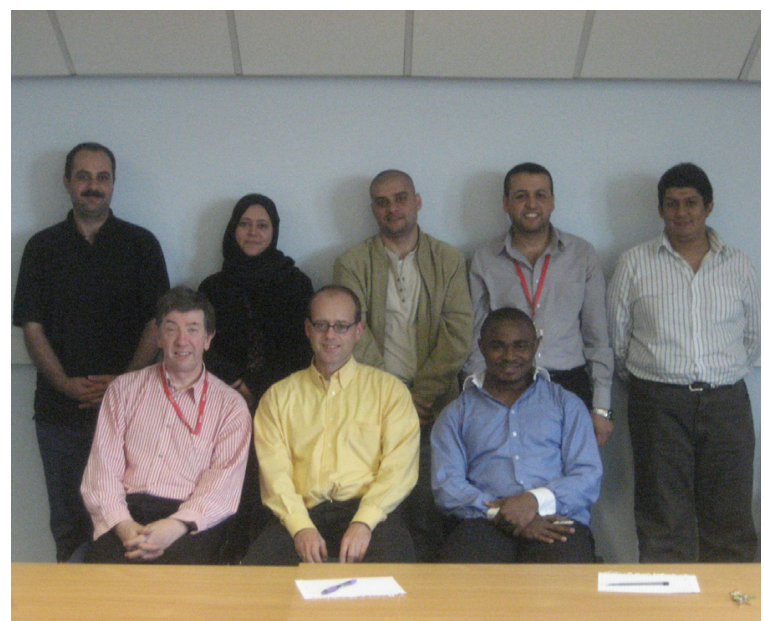

Jehad Al-Amri, Suad Almullah, Muneeb Dawood, Mohanad Halaweh, Osita Ibekwe, Raed Kareem Kanaan, Mick Phythian, Abdullah Al-Shery, Khaled Swesi, and Sarai Tangai are research students and members of the Information Society Doctoral Programme (ISDP). 